\title{
O USO DE INDICADORES PARA O DIAGNÓSTICO DA PRESTAÇÃO DE SERVIÇO DE COLETA E TRATAMENTO DO ESGOTO DOMÉSTICO NA CIDADE DE AQUIDAUANA/MS ${ }^{1}$
}

\author{
Ricardo Miranda dos Santos ${ }^{2}$
}

RESUMO: A finalidade dos indicadores é fornecer informações que permitam auxiliar os gerenciadores e administradores públicos na elaboração do planejamento e execução das políticas públicas, visando à melhoria da qualidade de vida. $\mathrm{O}$ uso de indicadores ambientais empregados por setores censitários possibilita a interação das mais diversas informações em um mesmo ambiente, ampliando as ações estratégias na tomada de decisão. As ações baseadas nas informações obtidas por meio dos Indicadores Ambientais, considerando as variáveis ambientais e antrópicas são capazes de demonstrar seu desempenho ao longo do tempo, e de realizar previsões. A presente pesquisa foi realizada na área urbana do município de Aquidauana/MS/BRASIL visando avaliar e conhecer com maior detalhe o conjunto das condições propícias à saúde pública e os problemas de cada área da cidade. Para alcançar os objetivos da pesquisa utilizou-se o Indicador de Esgotamento Sanitário-les, que é composto pelas variáveis: Indicador de Cobertura em Coleta de Esgotos e Tanques Sépticos-Ice; Tratamento de Esgotos e Tanques Sépticos-Ite e Saturação do Tratamento-Ise, adaptado do modelo Indicador de Salubridade Ambiental-ISA que foi desenvolvido pelo Conselho Estadual de Saneamento do Estado de São Paulo, em 1999. Assim o objetivo da pesquisa é demonstrar como a utilização dos Indicadores Ambientais pode auxiliar o planejamento na obtenção de informações necessárias para orientar os administradores públicos a tomarem decisões e a diagnosticar a salubridade ambiental da área urbana.

Palavras-chave: Aquidauana/MS. Esgotamento Sanitário. Meio Ambiente.

\footnotetext{
${ }^{1}$ Este artigo contém parte dos resultados da dissertação de mestrado defendida em 2008, no campus de Aquidauana/MS pela UFMS, como bolsistas da FUNDECT/CAPES.

${ }^{2}$ Mestre em Geografia pela Universidade Federal de Mato Grosso do Sul - UFMS - Campus de Aquidauana/MS; Bolsista da FUNDECT/CAPES, Vinculado a Fundação para o Desenvolvimento da UNESP - FUNDUNESP, como Educador Online. E-mail: ricardomiranda4@hotmail.com
} 


\section{INTRODUÇÃO}

Nas últimas décadas o espaço urbano sofreu alterações devido a concentração populacional o que acarretou em um ambiente com baixa salubridade devido aos baixos investimentos em saneamento básico. Um exemplo deste quadro é a produção de esgotos nos centros urbanos, sendo que na maioria das cidades, grande quantidade de esgoto domiciliar não recebe nenhum tipo de tratamento.

E segundo a Organização Mundial da Saúde, "saneamento é o controle de todos os fatores do meio físico do homem, que exercem ou podem exercer efeitos nocivos sobre seu bem estar físico, mental e social" e a Organização Mundial da Saúde define também saúde como sendo o "estado de completo bem estar físico, mental ou social, e não somente a ausência de doenças" (BARROS et al., 1995, p. 13).

Desta forma podemos entender que "saneamento é um conjunto de ações sobre o meio ambiente que controlam os fatores que possa provocar efeitos nocivos à saúde" promovendo um quadro de condições de salubridade ambiental. (SANTOS, 2008, p.18).

Assim, os municípios brasileiros devem procurar caminhos para superar tal problemática e para isso é necessário um sistema organizado de informações a fim de nortear o planejamento da cidade.

O conceito de planejamento e compreendido nas palavras de Almeida (et al. 1999, p. 123), planejamento pode ser visto como instrumento aplicável a vários tipos e níveis de atividade humana, sendo um instrumento direcionador para a racionalização na tomada de decisões individuais ou coletivas para se atingir um objetivo: "pode-se afirmar que o planejamento é a aplicação racional do conhecimento do homem ao processo e tomada de decisões para conseguir uma ótima utilização dos recursos, a fim de obter o máximo de benefícios para a coletividade".

Para Santos (2004) a estrutura do planejamento deve envolver pesquisa, análise e síntese, sendo que a pesquisa é compreendida pela organização e reunião de dados que facilite a sua interpretação. A análise avalia os dados organizados para compreender o meio estudado, com seus conflitos e acertos. A síntese é a aplicação do conhecimento 
alcançado para a tomada de decisões. Desta forma, o planejamento é elaborado em fases, onde o resultado de um é a base para o desenvolvimento da fase seguinte, sendo que cada fase tem componentes, métodos e produtos específicos.

O planejamento ambiental visa o desenvolvimento sustentável, proporcionando a melhoria da qualidade de vida e minimizando os impactos sobre a natureza e para tal, o levantamento de uma variedade de informações de diversas áreas do conhecimento se torna indispensável. É nesse sentido que o presente artigo visa abordar a aplicação dos indicadores ambientais como ferramenta de planejamento.

Os indicadores podem subsidiar e orientar os gestores públicos e a sociedade civil na tomar decisão e na busca pela melhoria da qualidade ambiental e de vida. Contudo compreendemos que para avaliar de forma efetiva a prestação dos serviços de coleta e tratamento de esgoto domiciliar da cidade de Aquidauana/MS envolve muitas interfases e fontes de dados, por este motivo não pretendemos findar a discussão sobre a situação do esgotamento sanitário do município, mas ser um ponto de partida para futuras discussões. $\mathrm{O}$ artigo procura discutir o uso dos indicadores como ferramenta para auxiliar os administradores públicos no planejamento e investimentos dos recursos financeiros, destacamos também a necessidade de agregar outros dados para chegar o mais próximo da realidade da situação do esgotamento sanitário do município.

Como afirma Santos (2004 p. 60):

\begin{abstract}
Os indicadores são fundamentais para tomadores de decisão e para a sociedade, pois permitem tanto criar cenários sobre o estado do meio, quanto aferir ou acompanhar os resultados de uma decisão tomada. São indicativos das mudanças e condições no ambiente e, se bem conduzidos permitem representar a rede de causalidades presentes num determinado meio. Os indicadores são empregados para avaliar e comparar territórios de diferentes dimensões e de diversas complexidades.
\end{abstract}

Assim, Junior Philippi (2005) afirma que uma das funções dos indicadores é apresentar de forma mais simples, transparente e acessível para a comunidade e gestores públicos a complexidade das questões existentes no processo de gestão dos ambientes naturais e antrópicos, objetivando conscientizar a todos do quadro socioeconômico e ambiental existente.

A área de estudo foi a malha urbana de Aquidauana/MS (Figura 01) localiza-se na porção centro-sudoeste do Estado de Mato Grosso do Sul, situado geograficamente entre 


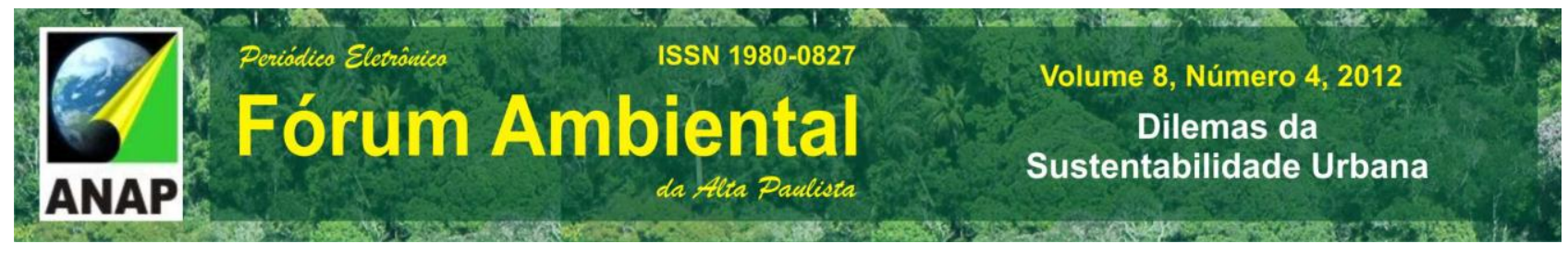

as latitudes $20^{\circ} 26^{\prime} 23^{\prime \prime S}$ e $20^{\circ} 29^{\prime} 21^{\prime \prime} S$ e de longitude $55^{\circ} 48^{\prime} 26^{\prime \prime}$ e $55^{\circ} 44^{\prime} 49^{\prime \prime}$ W; ficando a $130 \mathrm{Km}$ da capital Campo Grande (IBGE, 2005).

A área territorial do município é de 16.958,496 km2, Aquidauana é o $4^{\circ}$ maior município do Estado, possui uma densidade Demografia de 2,71 hab/km2, sendo $59^{\circ}$ maior densidade demográfica do Estado de MS, a estimativa da população 2005 é de 46.007 habitantes, a 6o maior população de MS. Sobre a taxa de urbanização, Aquidauana/MS posiciona-se como o $24^{\circ}$ lugar do Mato Grosso do Sul, sendo que $77,85 \%$ encontravam-se na zona urbana e $22,15 \%$ na zona rural (IBGE, 2005).

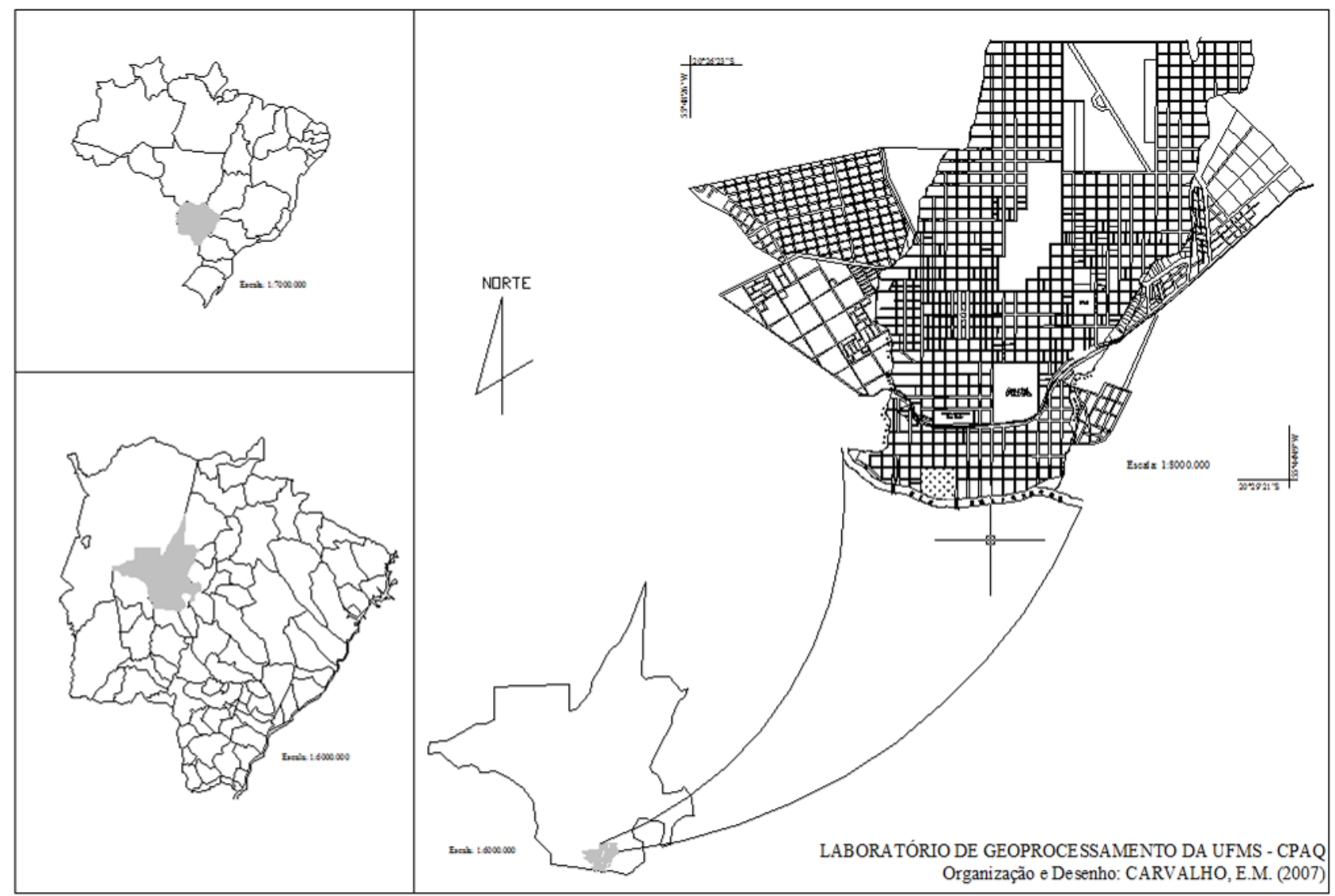

Figura 01 - Localização da área urbana de Aquidauana no Brasil, no Estado de Mato Grosso do Sul e na área total do município.

Fonte: SANTOS, 2008 p.21.

Para se analisar do sistema de esgotamento sanitário a pesquisa utilizou os setores censitários do Instituto Brasileiro de Geografia e Estatística - IBGE. O Município de Aquidauana/MS está dividido em 35 setores censitários (Figura 02) sendo à base de 
aplicação, análise e comparação do modelo de indicador adotado, desta forma permitindo visualizar as desigualdades e levantar os problemas locais em cada um deles.

O Indicador de Salubridade Ambiental - ISA e composto por vários sub-indicadores, e para esta pesquisa foi utilizado o Indicador de Esgotamento Sanitário - les, no qual permitirá realizar um estudo sobre o sistema de esgotamento sanitário do Município de Aquidauana/MS. O ISA é uma ferramenta de análise e avaliação criado pelo Conselho Estadual de Saneamento com a finalidade de desenvolver políticas públicas para a crescente melhora da qualidade de vida no Estado de São Paulo. (SÃO PAULO, 1999).

As informações que irão compor o Indicador de Esgotamento Sanitário serão levantadas pelo IBGE por setor censitário e outra fonte de pesquisa foi a Empresa de Saneamento do Mato Grosso do Sul S.A. - SANESUL.

Visando o auxilio ao processo decisório e possibilitando informações com diferencial agregado no valor das análises, os dados obtidos podem ser espacializados por meio de mapas temáticos para dos dados. Assim a utilização dos indicadores ambientais nos permite fazer sua aplicação dividindo a cidade em setores, demonstrando a realidade das condições sanitárias em cada um dessas unidades, mas as informações serão pontuais no tempo e no espaço, permitindo que o administrador público direcionar os recursos financeiros para cada problemática encontrada nestes setores. A qualidade de vida urbana se dá através de uma visão holística, visando à melhoria em cada um dessas unidades, objetivando a salubridade do ambiente urbano no seu todo.

\section{METODOLOGIA}

O Município de Aquidauana/MS possui 35 setores censitários (Figura 02) que foram a base de aplicação do Indicador de Esgotamento Sanitário. A informações que fizeram parte da composição das variáveis ou sub-indicadores deste indicador foram levantadas pelo IBGE por meio do software (Sistema de Recuperação de Informação Georreferenciadas - ESTATCART - Figura 03), que apresenta informações por setor censitário. 

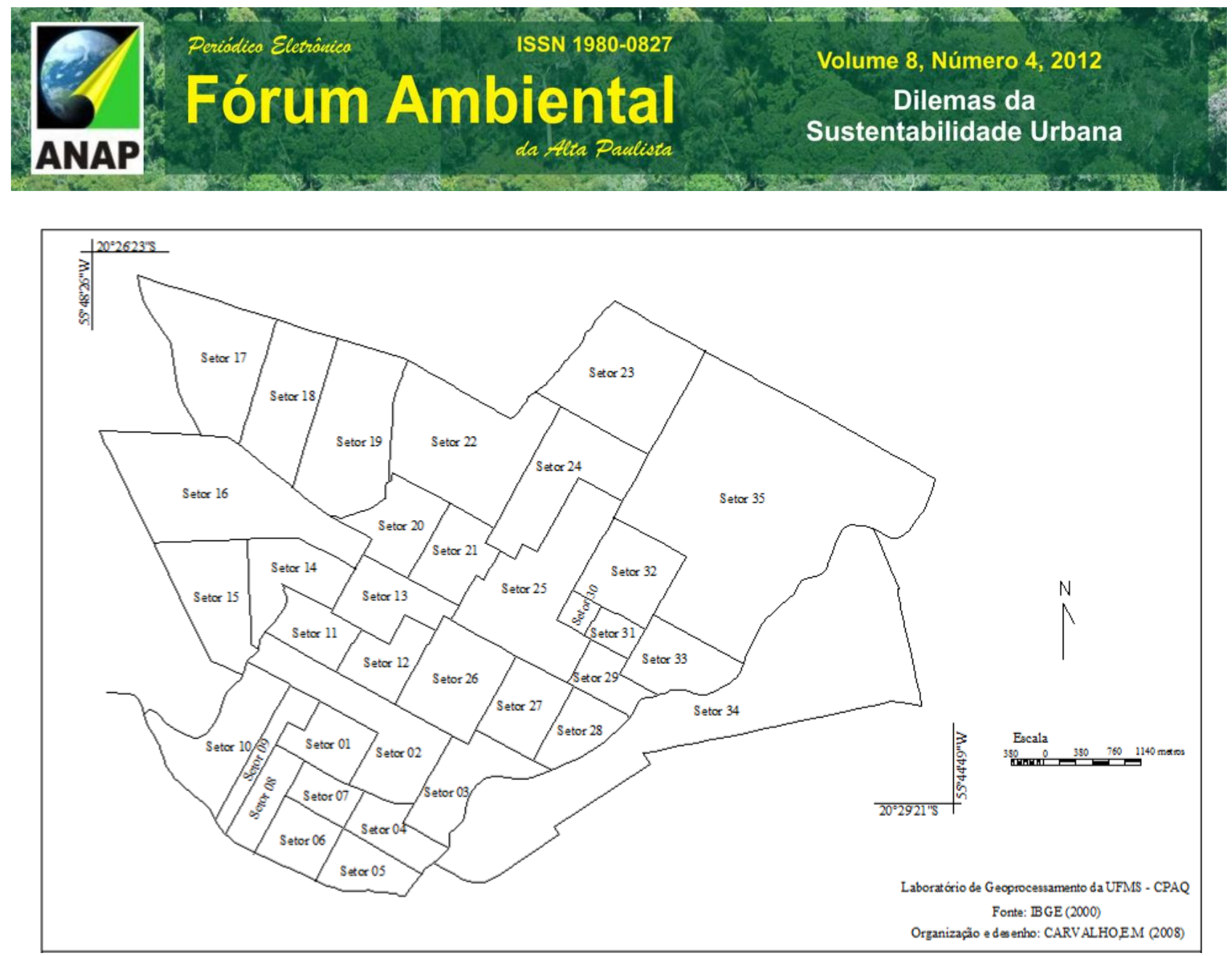

Figura 02 - Mapa de localização de setores censitários do IBGE (2000) da área urbana do Município de Aquidauana/MS.

Fonte: SANTOS, 2008 p.81.

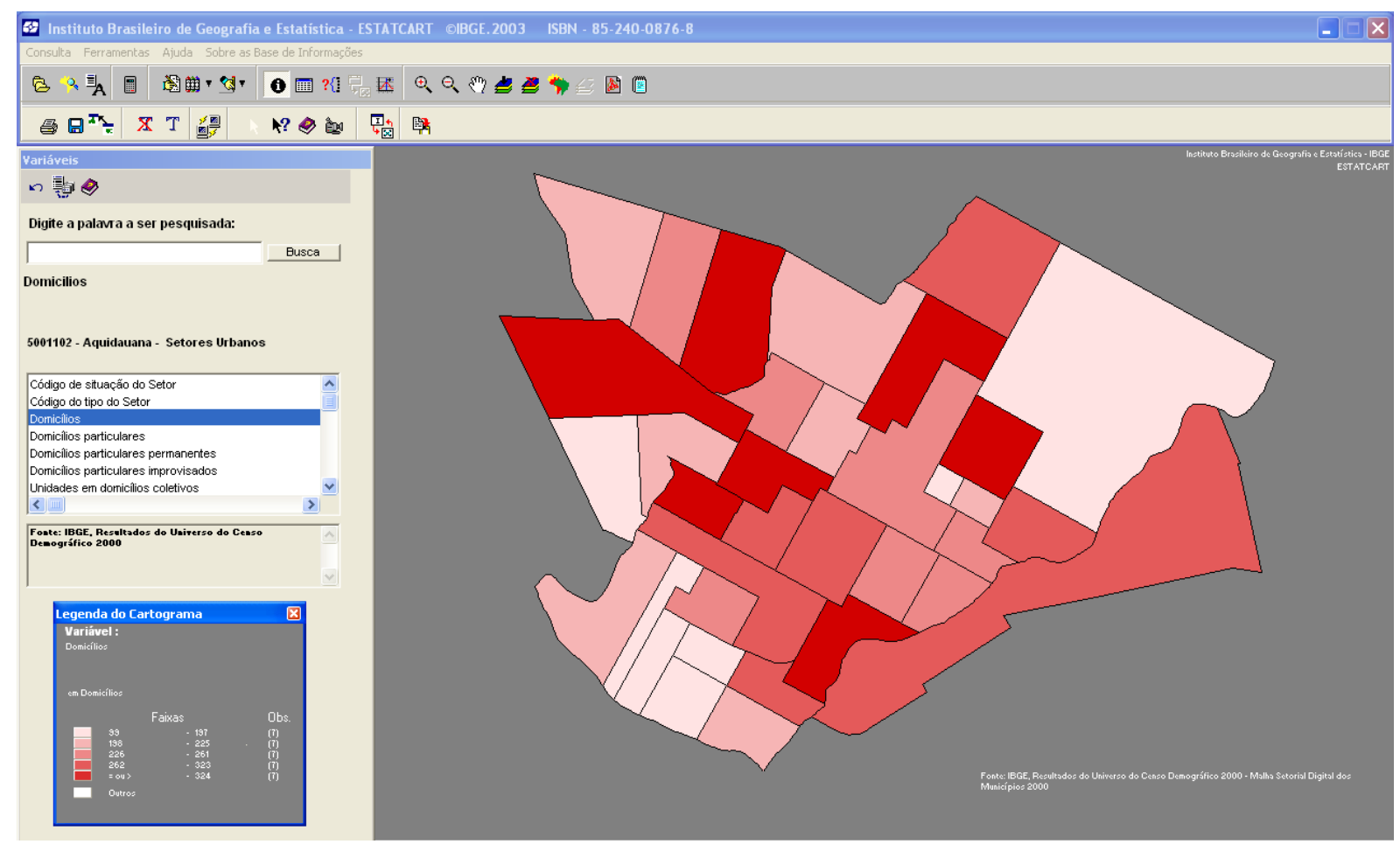

Figura 03: Visualização das informações por setor censitário do software do IBGE - ESTATCART Fonte: SANTOS, 2008 p.80. 


\subsection{Indicador de Esgotamento Sanitário - les}

O Indicador de Esgototamento Sanitário - les é calculado pela soma entre os Ice (Indicador de Cobertura em Coleta de Esgotos e Tanques Sépticos), Ite (Tratamento de Esgotos e Tanques Sépticos) e Ise (Saturação do Tratamento), dividido por três (Quadro 01) (SÃO PAULO, 1999).

Quadro 01. Critério de Aplicação do Indicador de Esgotamento Sanitário - les

\begin{tabular}{|c|c|c|}
\hline \multirow{2}{*}{$\begin{array}{l}\text { Indicador de cobertura de } \\
\text { coleta de esgoto } \\
\text { Ice }\end{array}$} & $\begin{array}{l}\text { DUE = Domicílios urbanos atendidos por coleta mais } \\
\text { tanques sépticos. }\end{array}$ & \multirow{2}{*}{ Ice $=\frac{\text { DUE }}{\text { DUT }} \times 100$} \\
\hline & DUT 0 Domicilio urbano totais & \\
\hline \multirow{3}{*}{$\begin{array}{l}\text { Indicador de esgotos } \\
\text { tratados e tanques } \\
\text { sépticos } \\
\text { Ite }\end{array}$} & Ice = Índice de cobertura de esgoto & \multirow{3}{*}{ Ite $=$ ice $\times \frac{\mathrm{VT}}{\mathrm{VC}} \times 100$} \\
\hline & $\begin{array}{l}\mathrm{VT}=\text { Volume tratado de esgotos medido ou estimado } \\
\text { nas estações em áreas servidas por rede esgoto. }\end{array}$ & \\
\hline & $\mathrm{VC}=$ Volume coletado de esgoto & \\
\hline \multirow{4}{*}{$\begin{array}{l}\text { Indicador de saturação } \\
\text { do tratamento de esgoto } \\
\text { Ise }\end{array}$} & $\begin{array}{l}\mathrm{N}=\text { Número de anos em que o sistema ficará } \\
\text { saturado }\end{array}$ & \multirow{3}{*}{$\log \frac{C T}{V}$} \\
\hline & $\mathrm{VC}=$ Volume coletado de esgotos & \\
\hline & CT = Capacidade de tratamento & \\
\hline & $\begin{array}{l}\mathrm{t}=\text { Taxa de crescimento anual ou médio da } \\
\text { população urbana para os } 5 \text { anos subseqüentes ao } \\
\text { ano de elaboração do ISA (projeção). }\end{array}$ & $\log (1+t)$ \\
\hline
\end{tabular}

Fonte: Adaptado pelo autor a partir de São Paulo (1999).

O Indicador de Cobertura e de Coleta de Esgotos e Tanques Sépticos possui sua pontuação variando entre 0 (zero) e 100 (cem), sendo os seus resultados analisados de acordo com a faixa populacional (Quadro 02 e 03). O período de atualização é a cada seis meses correspondentes aos meses de junho a dezembro (SÃO PAULO, 1999).

Quadro 02. Faixa populacional para classificação do Indicador de Cobertura de Coleta de Esgotos - Ice

\begin{tabular}{|l|c|c|}
\hline $\begin{array}{c}\text { Faixas de classificação dos } \\
\text { municípios referente á } \\
\text { População }\end{array}$ & Mínimo & Máximo \\
\hline Até 5.000 hab. & Ice $<50 \%-$ Ice $=0$ & Ice $>85 \%-$ Ice $=100$ \\
\hline de 5.000 a 20.000 hab. & Ice $<55 \%-$ Ice $=0$ & Ice $>85 \%-$ Ice $=100$ \\
\hline de 20.000 a 50.000 hab. & Ice $<60 \%-$ Ice $=0$ & Ice $>85 \%-$ Ice $=100$ \\
\hline de 50.000 a 100.000 hab. & Ice $<65 \%-$ Ice $=0$ & Ice $>85 \%-$ Ice $=100$ \\
\hline de 100.000 a 500.000 hab. & Ice $<70 \%-$ Ice $=0$ & Ice $>90 \%-$ Ice $=100$ \\
\hline$>500.000$ hab. & Ice $<75 \%-$ Ice $=0$ & Ice $>90 \%-$ Ice $=100$ \\
\hline
\end{tabular}

Fonte: Adaptado pelo autor a partir de São Paulo (1999). 
O Indicador de Esgoto Tratado - Ite tem como objetivo quantificar os domicílios atendidos por tratamento de esgotos e tanques sépticos, seu período de atualização e sequencial, sempre que houver modificações de atendimento. (SÃO PAULO, 1999).

Quadro 03. Faixa populacional para classificação do Indicador de Esgotos Tratados e Tanques Sépticos - Ite

\begin{tabular}{|c|c|c|}
\hline $\begin{array}{c}\text { Faixas de classificação dos } \\
\text { municípios referente á } \\
\text { População }\end{array}$ & Mínimo & Máximo \\
\hline Até 5.000 hab. & Ite $<15,00 \%$ - Ite $=0$ & Ite $>56,00 \%-$ Ite $=100$ \\
\hline de 5.000 a 20.000 hab. & Ite $<16,50 \%-$ Ite $=0$ & Ite $>63,75 \%$ - Ite $=100$ \\
\hline de 20.000 a 50.000 hab. & Ite $<18,00 \%$ - Ite $=0$ & Ite $>68,00 \%-$ Ite $=100$ \\
\hline de 50.000 a 100.000 hab. & Ite $<26,00 \%-$ Ite $=0$ & Ite $>72,25 \%-$ Ite $=100$ \\
\hline de 100.000 a 500.000 hab. & Ite $<35,00 \%-$ Ite $=0$ & Ite $>81,00 \%-$ Ite $=100$ \\
\hline$>500.000$ hab. & Ite $<45,00 \%-$ Ite $=0$ & Ite $>81,00 \%-$ Ite $=100$ \\
\hline
\end{tabular}

Fonte: Adaptado pelo autor a partir de São Paulo (1999).

O Indicador de Saturação do Tratamento de Esgoto - Ise possui a finalidade de comparar a oferta e demanda das instalações existentes e programar novas instalações ou ampliações. O período de atualização é anual no mês de dezembro ou quando houver expansão, ampliação ou construção de novos sistemas de tratamento e/ou eventuais correções ou atualização de cadastros. O resultado obtido por meio de cálculos é aplicado de acordo com a faixa populacional (quadro 04) (SÃO PAULO, 1999).

Quadro 04. Faixa populacional do Indicador de Saturação do Tratamento de Esgotos - Ise

\begin{tabular}{|c|c|c|}
\hline FAIXAS DE POPULAÇÃO & $\mathbf{n}^{*}$ & 100 \\
\hline \multirow{3}{*}{ até 50.000 hab. } & $\mathrm{n}>=2$ & 50 \\
\cline { 2 - 3 } & $2>\mathrm{n}>0$ & 0 \\
\cline { 2 - 3 } & $\mathrm{n}<=0$ & 100 \\
\cline { 2 - 3 } de 50.000 a 200.000 hab. & $\mathrm{n}>=3$ & 50 \\
\cline { 2 - 3 } & $\mathrm{n}>\mathrm{n}>0$ & 100 \\
\cline { 2 - 3 } & $\mathrm{n}>=0$ & 50 \\
\cline { 2 - 3 } & $5>\mathrm{n}>0$ & 0 \\
\hline \multirow{2}{*}{ maior de 200.000 hab. } & $\mathrm{n}<=0$ & 0 \\
\hline
\end{tabular}

$\mathrm{n}^{*}=$ resultado obtido por meio da formula do indicador que será empregado de acordo com o número populacional do município.

Fonte: Adaptado pelo autor de São Paulo (1999). 


\section{RESULTADOS E DISCUSSÃO}

O Indicador de Esgotamento Sanitário - les tem como objetivo avaliar a condição da disposição do esgoto nas residências por setores. A utilização do indicador pode auxiliar na melhoria da qualidade ambiental e direcionar os recursos financeiros para a ampliação do sistema de coleta e tratamento de esgoto na área urbana.

A finalidade do Indicador de Cobertura de Coleta de Esgoto - Ice é quantificar os domicílios atendidos por rede de esgoto e/ou tanques sépticos. Para gerar este indicador foram utilizados os dados do Censo Demográfico de 2000 quanto aos domicílios atendidos pela rede geral ou pluvial ou por tanques ou fossas sépticas. A cobertura da rede de esgoto abrange uma pequena porção do espaço urbano da cidade de Aquidauana (Figura 04), onde se apresenta a localização da rede de esgoto do Município de Aquidauana-MS (SANESUL, 2007). Na área urbana de Aquidauana, a maioria dos setores utiliza outros tipos de sistemas de esgotamento sanitário, como por exemplo, a fossa séptica.

A cidade de Aquidauana não apresentou nenhum setor com 100\% de domicílios atendidos pelo sistema coletor da rede geral ou por fossa séptica. Apenas os setores 04, 06, 07, 09 e 10 ficaram acima dos $90 \%$ de atendimento. Os setores $01,02,05,08,32$ e 33 entre $50 \%$ a $90 \%$, e na grande maioria os setores ficaram abaixo de $5 \%$ de domicílios atendidos.

A cidade de Aquidauana não apresentou nenhum setor com 100\% de domicílios atendidos pelo sistema coletor da rede geral ou por fossa séptica. Apenas os setores 04, 06, 07, 09 e 10 ficaram acima dos $90 \%$ de atendimento. Os setores $01,02,05,08,32$ e 33 entre $50 \%$ a $90 \%$, e na grande maioria os setores ficaram abaixo de $5 \%$ de domicílios atendidos. 

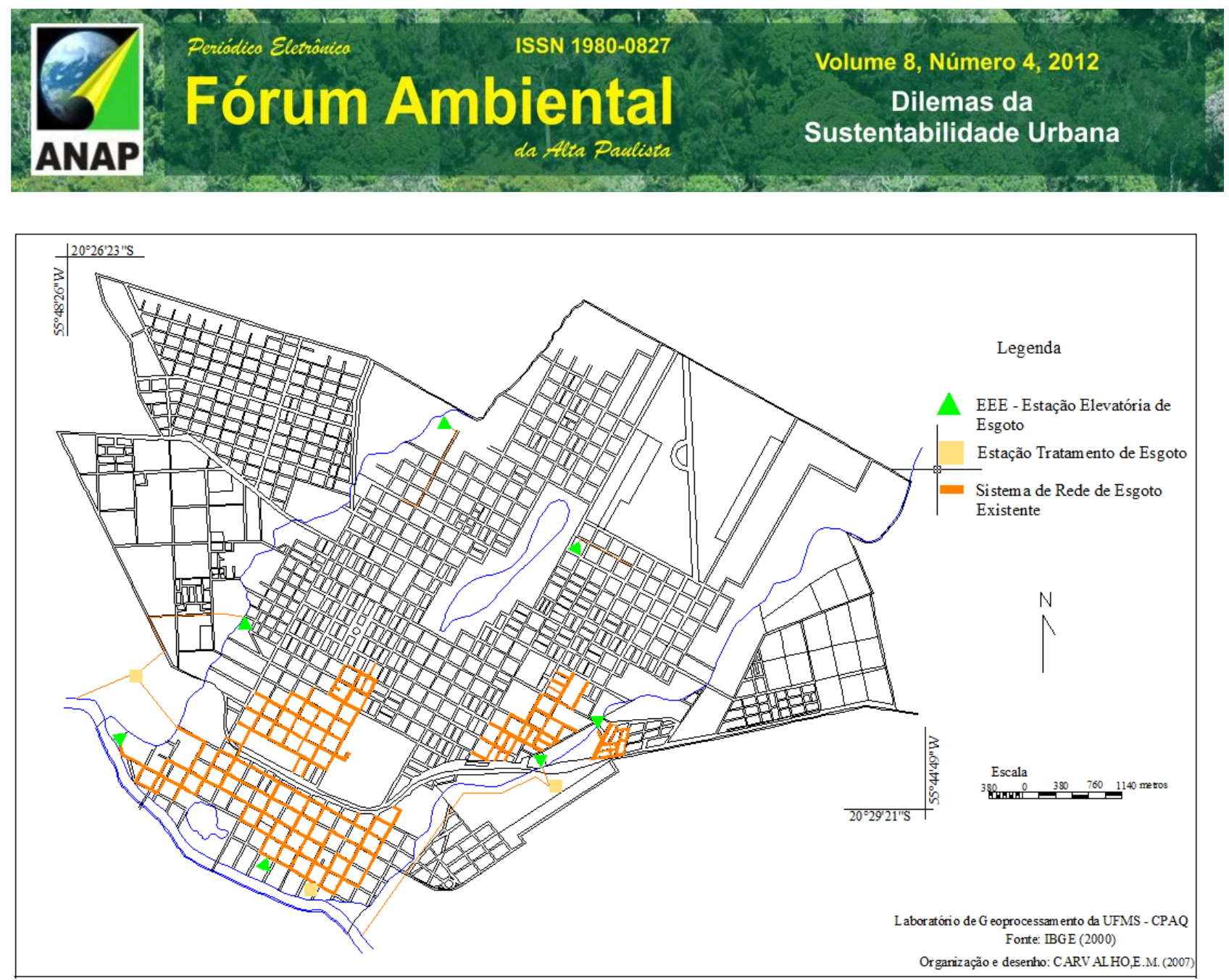

Figura 04: Mapa de localização da rede de coleta e estações de tratamento de esgoto da área urbana de Aquidauana-MS.

Fonte: SANTOS, 2008 p.111.

A cidade de Aquidauana não apresentou nenhum setor com 100\% de domicílios atendidos pelo sistema coletor da rede geral ou por fossa séptica. Apenas os setores 04, 06, 07, 09 e 10 ficaram acima dos 90\% de atendimento. Os setores 01, 02, 05, 08, 32 e 33 entre $50 \%$ a $90 \%$, e na grande maioria os setores ficaram abaixo de $5 \%$ de domicílios atendidos.

Ao analisar a relação com o mapa de rede geral de esgotamento sanitário (Figura 04), evidenciando uma incidência muito grande de domicílios ligados à rede geral, mas nem por isso ocorrem 100\% de ligações dos domicílios, os possíveis motivos são: o custo da taxa de esgoto, a fragilidade de legislação específica que discipline tal cenário e a ausência de campanhas de educação que demonstrem a importância e as vantagens á saúde pública. A tabela 01 estabelece uma relação em porcentagem dos setores que são atendidos por rede geral, por fossa séptica e outras formas de disposição do esgoto. As outras formas de esgotamento sanitário mencionadas na Tabela 01 referem-se à fossa rudimentar, vala, rio, ou outro tipo de escoadouro. Desta forma, a situação de esgotamento sanitário da área urbana de Aquidauana encontra-se preocupante, pois a 
maior parte dos setores possui porcentagem alta de domicílios atendidos por "outras formas" de esgotamento sanitário, comprometendo a qualidade do meio e colocando em risco a saúde da população (Tabela 02).

Tabela 01. Porcentagem de domicílios urbanos atendidos por rede geral, por fossa Séptica e por outras formas de destino do esgoto na área urbana do município de Aquidauana/MS.

\begin{tabular}{|c|c|c|c|c|c|c|c|}
\hline $\begin{array}{l}\text { ఖे } \\
\text { Фे } \\
\text { ஸे }\end{array}$ & $\begin{array}{l}\text { № de } \\
\text { Domicílios - } \\
\text { Rede Geral } \\
\%\end{array}$ & $\begin{array}{l}\text { № de } \\
\text { Domicílios - } \\
\text { Fossa } \\
\text { Séptica \% }\end{array}$ & $\begin{array}{l}\text { № de } \\
\text { Domicílios - } \\
\text { Outras } \\
\text { Formas \% }\end{array}$ & $\begin{array}{l}\text { ఖे } \\
\text { 흉 } \\
\text { ஸे }\end{array}$ & $\begin{array}{l}\text { № de } \\
\text { Domicílios - } \\
\text { Rede Geral } \\
\%\end{array}$ & $\begin{array}{l}\text { № de } \\
\text { Domicílios - } \\
\text { Fossa } \\
\text { Séptica \% }\end{array}$ & $\begin{array}{l}\text { № de } \\
\text { Domicílios - } \\
\text { Outras } \\
\text { Formas \% }\end{array}$ \\
\hline 01 & 75 & 7 & 18 & 19 & 0 & 0 & 100 \\
\hline 02 & 55 & 10 & 35 & 20 & 0 & 0 & 100 \\
\hline 03 & 12 & 1 & 87 & 21 & 0,5 & 0,5 & 99 \\
\hline 04 & 85 & 13 & 2 & 22 & 0 & 4 & 96 \\
\hline 05 & 48 & 39 & 13 & 23 & 0 & 0 & 100 \\
\hline 06 & 62 & 32 & 6 & 24 & 0 & 0 & 100 \\
\hline 07 & 87 & 7 & 6 & 25 & 1 & 25 & 74 \\
\hline 08 & 78 & 1 & 21 & 26 & 2 & 0 & 98 \\
\hline 09 & 88 & 9 & 3 & 27 & 3 & 1 & 96 \\
\hline 10 & 57 & 36 & 7 & 28 & 5 & 0 & 95 \\
\hline 11 & 31 & 3 & 66 & 29 & 0 & 0 & 100 \\
\hline 12 & 19 & 4 & 77 & 30 & 0 & 2 & 98 \\
\hline 13 & 9 & 1 & 90 & 31 & 3 & 3 & 94 \\
\hline 14 & 0,5 & 0,5 & 99 & 32 & 0 & 62 & 38 \\
\hline 15 & 0 & 2 & 98 & 33 & 0 & 51 & 49 \\
\hline 16 & 0 & 46 & 54 & 34 & 0 & 1 & 99 \\
\hline 17 & 0 & 0 & 100 & 35 & 0 & 0 & 100 \\
\hline 18 & 0 & 1 & 99 & & & & \\
\hline
\end{tabular}

Fonte: Censo Demográfico de 2000 (IBGE, 2004).

Tabela 02. Pontuação de acordo com a faixa populacional do Indicador de Cobertura de Coleta de Esgoto - Ice

\begin{tabular}{|c|c|c|c|c|c|}
\hline Setores & Ice $\%$ & $\begin{array}{l}\text { Pontuação referente à } \\
\text { faixa populacional }^{\star}\end{array}$ & Setores & Ice \% & $\begin{array}{l}\text { Pontuação referente à } \\
\text { faixa populacional }{ }^{\star}\end{array}$ \\
\hline 01 & 82,35 & 50 & 19 & 0 & 0 \\
\hline 02 & 64,94 & 50 & 20 & 0 & 0 \\
\hline 03 & 12,96 & 0 & 21 & 0,92 & 0 \\
\hline 04 & 98,09 & 100 & 22 & 4,07 & 0 \\
\hline 05 & 86,87 & 100 & 23 & 0 & 0 \\
\hline 06 & 94,41 & 100 & 24 & 0,23 & 0 \\
\hline 07 & 94,02 & 100 & 25 & 25,71 & 0 \\
\hline 08 & 78,78 & 50 & 26 & 1,74 & 0 \\
\hline 09 & 97,24 & 100 & 27 & 3,62 & 0 \\
\hline 10 & 92,72 & 100 & 28 & 5,30 & 0 \\
\hline 11 & 33,02 & 0 & 29 & 0 & 0 \\
\hline 12 & 23,31 & 0 & 30 & 1,29 & 0 \\
\hline 13 & 9,84 & 0 & 31 & 5,45 & 0 \\
\hline 14 & 0,90 & 0 & 32 & 62,46 & 50 \\
\hline
\end{tabular}




\begin{tabular}{c|c|c|c|c|c}
\hline $\mathbf{1 5}$ & 1,68 & 0 & $\mathbf{3 3}$ & 50,85 & 0 \\
\hline $\mathbf{1 6}$ & 46,50 & 0 & $\mathbf{3 4}$ & 0,61 & 0 \\
\hline $\mathbf{1 7}$ & 0 & 0 & $\mathbf{3 5}$ & 0 & 0 \\
\hline $\mathbf{1 8}$ & 1,3 & 0 & & & \\
\hline
\end{tabular}

*Segundo o Manual do ISA o resultado do Indicador de Cobertura e Coleta de Esgotos é submetido a uma tabela (vide metodologia) que divide os municípios por densidade populacional e estabelece pontuações mínimas e máximas. Como o município de Aquidauana possui uma população dentro da faixa de 20.000 a 50.000 habitantes, o índice de cobertura de coleta de esgoto deve ser superior a $85 \%$ para receber 100 pontos, entre $60 \%$ a $85 \%$ pontuação de 50 pontos e inferior a $60 \%$ recebe 0 pontos.

Fonte: SANTOS, 2008 p.114.

\subsection{Indicador de Esgotos Tratados - Ite}

A finalidade do Indicador de Esgoto Tratado é quantificar os domicílios atendidos por tratamento de esgotos. A disposição inadequada dos esgotos pode disseminar doenças como diarreia, verminose, teníase, esquistossomose e cólera, entre outras doenças. As ausências de condicionamento adequado, do afastamento e do tratamento dos esgotos, quanto associada a outros fatores como lixo e inundações resultam em altos índices de morbidade e mortalidade e a proliferação de insetos, roedores e outros vetores.

Os dados que compõem esse indicador são: volume coletado de esgoto e volume tratado de esgoto medido ou estimado na estação, em áreas servidas por rede de esgoto. De acordo com as informações adquiridas por meio do questionário aplicado a empresa de saneamento do município, o volume coletado de esgoto no mês de junho de 2007 foi de $12.156,80 \mathrm{~m}^{3}$. Deste total de esgoto coletado das áreas servidas por rede de esgoto, a empresa trata $100 \%$.

Ao calcular o consumo da média mensal de água, pode-se chegar à porcentagem aproximada do coeficiente de retorno ${ }^{3}$, ou seja, o total de esgoto produzido na área urbana de Aquidauana. Desta forma, a porcentagem aproximada do total de esgoto coletado e tratado do município ficou em aproximadamente 9,32\%. O cálculo do coeficiente de retorno não considerou as fossas sépticas, pois segundo a empresa de saneamento, nenhuma empresa especializada em limpar fossas a procura para tratar o

\footnotetext{
${ }^{3}$ De acordo com Barros (et. al. 1995, p.116) a vazão domestica de esgotos é calculada com base no consumo de água da localidade, ou seja em função do consumo médio diário de água de um individuo, denominado Quota Per Capita (QPC). Calcula-se a vazão de esgotos utilizando-se o conceito de Coeficiente de Retorno. Tal coeficiente situa-se em torno de $80 \%$, ou seja, para cada $100 \mathrm{~L}$ de água consumida, são lançados aproximadamente $80 \mathrm{~L}$ de esgotos na rede de coleta.
} 
esgoto coletado. Para calcular o sub-indicador de esgoto tratado e tanques sépticos utilizou-se o resultado do sub-indicador de cobertura de coleta de esgoto - Ice, porém o Ice utiliza dados tanto de domicílios urbanos atendidos por coleta de rede geral como de fossa séptica. Assim, são apresentados na Tabela 03 os resultados do sub-indicador de esgotos tratados, que já se encontram relacionados com a classificação dos municípios referente ao número de habitantes recomenda pelo manual do ISA, sendo que apenas 5 setores obtiveram pontuação de 100 pontos, relacionada a cobertura superior a $68 \%, 5$ setores apresentaram pontuação de 50\% referente a uma cobertura superior a $18 \%$, e 25 setores ficaram com pontuação 0 ou cobertura inferior a $18 \%$.

Tabela 03. Resultado do Indicador de Esgotos Tratados para os setores censitários a área urbana do município de Aquidauana/MS

\begin{tabular}{|c|c|c|c|c|c|}
\hline Setores & Ite por Setor & $\begin{array}{c}\text { Pontuação por Faixa } \\
\text { Populacional }^{*}\end{array}$ & Setores & Ite por Setor & $\begin{array}{c}\text { Pontuação por Faixa } \\
\text { Populacional }^{\star}\end{array}$ \\
\hline 01 & 74,78 & 100 & 19 & 0 & 0 \\
\hline 02 & 53,87 & 50 & 20 & 0 & 0 \\
\hline 03 & 12,34 & 0 & 21 & 0,46 & 0 \\
\hline 04 & 85,49 & 100 & 22 & 0,45 & 0 \\
\hline 05 & 47,51 & 50 & 23 & 0 & 0 \\
\hline 06 & 62,56 & 50 & 24 & 0,23 & 0 \\
\hline 07 & 86,95 & 100 & 25 & 1,22 & 0 \\
\hline 08 & 77,78 & 100 & 26 & 1,40 & 0 \\
\hline 09 & 88,28 & 100 & 27 & 2,42 & 0 \\
\hline 10 & 56,82 & 50 & 28 & 4,87 & 0 \\
\hline 11 & 11,93 & 0 & 29 & 0 & 0 \\
\hline 12 & 18,92 & 50 & 30 & 0 & 0 \\
\hline 13 & 8,92 & 0 & 31 & 2,72 & 0 \\
\hline 14 & 0,45 & 0 & 32 & 0,27 & 0 \\
\hline 15 & 0 & 0 & 33 & 0,34 & 0 \\
\hline 16 & 0,3 & 0 & 34 & 0,31 & 0 \\
\hline 17 & 0 & 0 & 35 & 0 & 0 \\
\hline 18 & 0,87 & 0 & & & \\
\hline
\end{tabular}

*Segundo o Manual do ISA o resultado do Indicador de Esgotos Tratados é submetido a uma tabela (vide metodologia), que divide os municípios por densidade populacional e estabelece pontuações mínimas e máximas. Como o município de Aquidauana possui uma população dentro da faixa de 20.000 a 50.000 habitantes o índice de cobertura deve ser superior a $68 \%$ para receber 100 pontos, entre $18 \%$ a $68 \%$ pontuação de 50 pontos e inferior a $18 \%$ recebe 0 pontos.

Fonte: SANTOS, 2008 p.116. 


\subsection{Indicador de Saturação do Tratamento de Esgoto - Ise}

A finalidade deste indicador é avaliar a oferta e a demanda das instalações existentes quanto ao tratamento de esgotos, visando programar novas instalações ou ampliações, sendo de extrema importância para o planejamento. Os dados que compõem o Indicador de Saturação do Tratamento de Esgoto são: capacidade de tratamento, volume coletado de esgoto e taxa de crescimento anual médio da população urbana para os próximos cinco anos. Segundo as informações obtidas pela empresa de saneamento a capacidade de tratamento da estação é de $40 \mathrm{~L} / \mathrm{s}$ em uma média de 15 horas por dia. $O$ volume de esgoto coletado e tratado no mês de junho de 2007 foi de $12.156,80 \mathrm{~m}^{3}$. Ao aplicar os dados na fórmula chegou se no valor 1,95 que foi analisado e aplicado em outro quadro de informações (vide metodologia) segundo a faixa populacional que pertence 0 município de Aquidauana. Como o município possui menos de 50.000 habitantes e o valor obtido ficou entre 0 e 2 o seu resultado deste indicador é de 50 pontos. Como a estação de tratamento atende todo o município, não é possível calcular o seu resultado por setores.

\subsection{Resultado final do Indicador de Esgotos Sanitário - les}

Com todos os resultados dos sub-indicadores calculados, pode-se chegar ao resultado do Indicador de Esgotos Sanitário - Ice e que é apresentado na Tabela 04 por setor.

Tabela 04. Resultado do Indicador de Esgotos Sanitário - les para os setores censitários da área urbana do município de Aquidauana/MS.

\begin{tabular}{|c|c|c|c|c|}
\hline Setores & $\begin{array}{c}\text { Indicador de } \\
\text { Cobertura em } \\
\text { coleta de esgoto - } \\
\text { Ice }\end{array}$ & $\begin{array}{c}\text { Indicador de } \\
\text { esgotos tratados } \\
\text { e tanques } \\
\text { Sépticos Ite }\end{array}$ & $\begin{array}{c}\text { Indicador de } \\
\text { Saturação do } \\
\text { Tratamento de } \\
\text { Esgoto - Ise }\end{array}$ & $\begin{array}{c}\text { Indicador de } \\
\text { Esgotos Sanitário } \\
\text { - les }\end{array}$ \\
\hline $\mathbf{0 1}$ & 82,35 & 74,78 & 50 & 69,04 \\
\hline $\mathbf{0 2}$ & 64,94 & 53,87 & 50 & 56,27 \\
\hline $\mathbf{0 3}$ & 12,96 & 12,34 & 50 & 25,10 \\
\hline $\mathbf{0 4}$ & 98,09 & 85,49 & 50 & 77,86 \\
\hline $\mathbf{0 5}$ & 86,87 & 47,51 & 50 & 61,46 \\
\hline $\mathbf{0 6}$ & 94,41 & 62,56 & 50 & 68,99 \\
\hline $\mathbf{0 7}$ & 94,02 & 86,95 & 50 & 76,99 \\
\hline
\end{tabular}




\begin{tabular}{|l|c|c|c|c|}
\hline $\mathbf{0 8}$ & 78,78 & 77,78 & 50 & 68,85 \\
\hline $\mathbf{0 9}$ & 97,24 & 88,28 & 50 & 78,50 \\
\hline $\mathbf{1 0}$ & 92,72 & 56,82 & 50 & 66,51 \\
\hline $\mathbf{1 1}$ & 33,02 & 11,93 & 50 & 31,65 \\
\hline $\mathbf{1 2}$ & 23,31 & 18,92 & 50 & 30,74 \\
\hline $\mathbf{1 4}$ & 9,84 & 8,92 & 50 & 22,92 \\
\hline $\mathbf{1 5}$ & 0,90 & 0,45 & 50 & 17,11 \\
\hline $\mathbf{1 6}$ & 1,68 & 0 & 50 & 17,22 \\
\hline $\mathbf{1 7}$ & 46,50 & 0,3 & 50 & 32,26 \\
\hline $\mathbf{1 8}$ & 0 & 0 & 50 & 16,66 \\
\hline $\mathbf{1 9}$ & 1,3 & 0,87 & 50 & 17,39 \\
\hline $\mathbf{2 0}$ & 0 & 0 & 50 & 16,66 \\
\hline $\mathbf{2 1}$ & 0 & 0 & 50 & 16,66 \\
\hline $\mathbf{2 2}$ & 0,92 & 0,46 & 50 & 17,13 \\
\hline $\mathbf{2 3}$ & 4,07 & 0,45 & 50 & 18,17 \\
\hline $\mathbf{2 4}$ & 0 & 0 & 50 & 16,67 \\
\hline $\mathbf{2 5}$ & 0,23 & 0,23 & 50 & 25,64 \\
\hline $\mathbf{2 6}$ & 25,71 & 1,22 & 50 & 17,71 \\
\hline $\mathbf{2 7}$ & 1,74 & 1,40 & 50 & 18,68 \\
\hline $\mathbf{2 8}$ & 3,62 & 2,42 & 50 & 20,06 \\
\hline $\mathbf{2 9}$ & 5,30 & 4,87 & 50 & 16,67 \\
\hline $\mathbf{3 0}$ & 0 & 0 & 50 & 17,10 \\
\hline $\mathbf{3 1}$ & 1,29 & 0 & 50 & 18,48 \\
\hline $\mathbf{3 2}$ & 5,45 & 0 & 50 & 38,39 \\
\hline $\mathbf{3 3}$ & 62,46 & 2,72 & 50 & 16,98 \\
\hline $\mathbf{3 4}$ & 50,85 & 0,27 & 50 & \\
\hline $\mathbf{3 5}$ & 0,61 & 0,34 & 50 & \\
\hline $\mathbf{S A N}$ & 0 & 0,31 & & \\
\hline & & & 50 & \\
\hline
\end{tabular}

Fonte: SANTOS, 2008 p.117.

A espacialização do indicador de esgotamento sanitário (Figura 05) demonstra os resultados por setores, divididos em cinco categorias. A figura comprova uma irregularidade do serviço de esgotamento sanitário para a maioria dos setores de Aquidauana. 
Figura 05. Espacialização do Resultado do Indicador de Esgotos Sanitários da área urbana de Aquidauana/MS

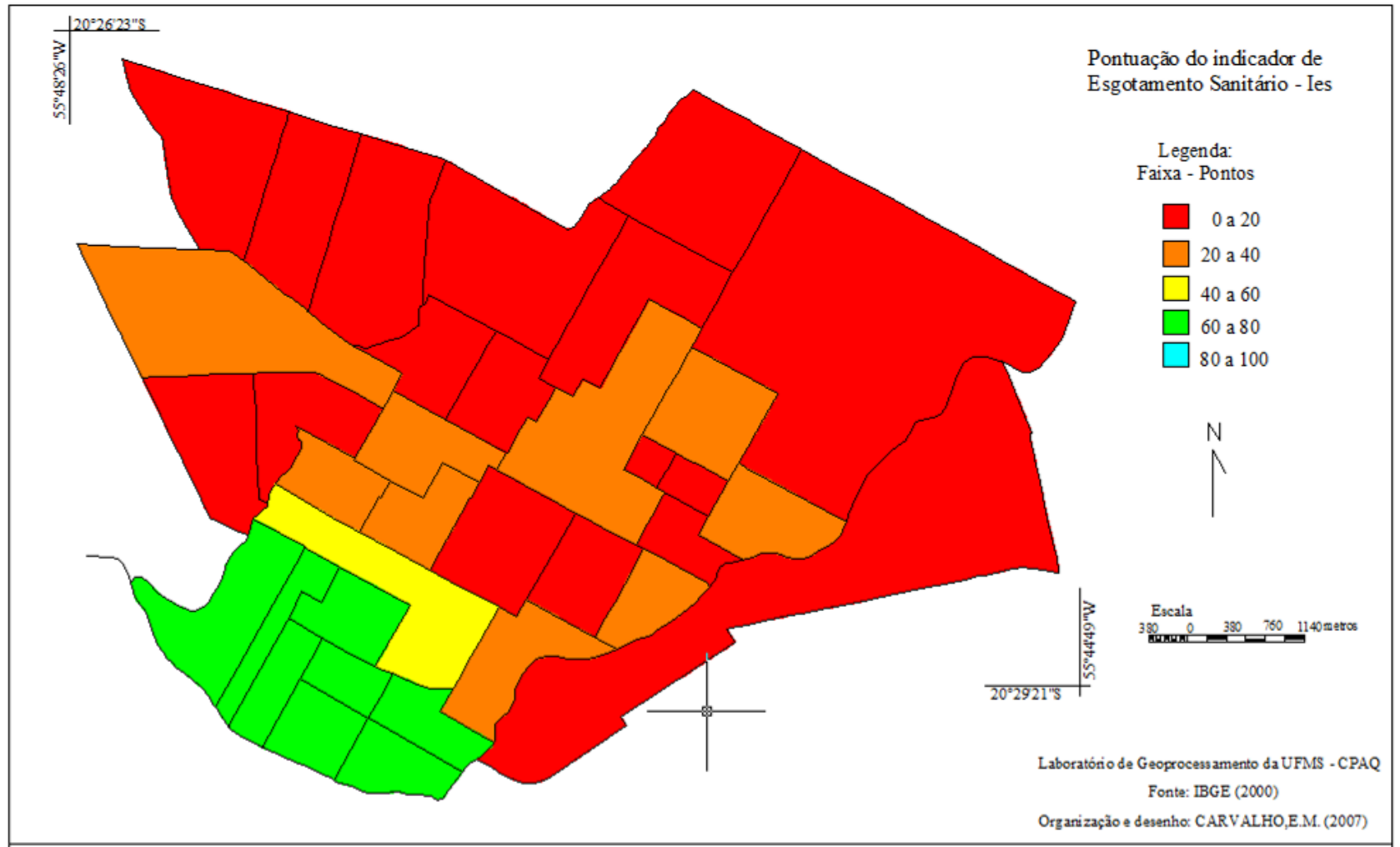

Fonte: SANTOS, 2008 p.117.

\section{CONCLUSÃO}

Atualmente existe um vasto banco de dados construídos por órgãos públicos federais, estaduais e municipais que estão à disposição de todos. Entretanto essa variedade de informação não é utilizada como deviria na elaboração de planejamentos. Em geral, a cada novo processo de planejamento, realiza-se nova etapa de levantamento de dados, gastando muito tempo, energia e dinheiro para a construção do próprio banco de dados, sendo que poder-se-ia utilizar as informações e dados já levantados de outras fontes e, desta forma, desprender mais tempo e energia na concretização das ações.

O sub-indicador de Cobertura de Coleta de Esgoto mostrou que a rede de esgoto abrange uma pequena porção do espaço urbano da cidade de Aquidauana. A situação do esgotamento sanitário de Aquidauana possui um quadro preocupante, pois possui 
porcentagem alta de domicílios atendidos por outras formas de esgotamento sanitário que colocando em risco a saúde da população. O sub-indicador de Esgotos Tratados revelou que somente $9,32 \%$ do total de esgoto é coletado e tratado. Este sub-indicador apresentou seu resultado único para todos os setores, sendo que, apenas os setores censitários que são atendidos por rede geral de cobertura do esgoto recebem tratamento.

O uso de indicadores demonstrou ser um instrumento adequado, de fácil aplicabilidade e de custo baixo, que incorpora uma grande quantidade de dados disponíveis de instituições, departamentos, secretarias e demais organizações para a aplicação do ISA ou de outros indicadores, sendo que tais informações estão disponíveis de forma gratuita. Entretanto a principal dificuldade do trabalho foi encontrar os dados disponíveis e organizadas em nível municipal.

Outra dificuldade encontra foi que os dados utilizados nesta pesquisa são do censo demográfico de 2000, a necessidade de informações mais atualizadas é imprescindível para representar com eficiência a realidade. Portanto, para que haja uma efetiva avaliação e monitoramento da salubridade ambiental do município, a administração pública municipal deve possuir um banco de dados organizado por setores viabilizando a sua comparação e acompanhando o desenvolvimento das ações desenvolvidas na cidade.

\section{REFERENCIAL}

ALMEIDA; J. R. [et al]. Planejamento Ambiental: caminho para participação popular e gestão ambiental para nosso futuro comum: uma necessidade, um desafio. $2^{\circ}$ Ed. Rio de Janeiro: THEX Ed.: Biblioteca Estácio de Sá. 1999.

BARROS, R. T. V; HELLER, L.; COSTA, A. M. L. M. Saneamento e o Município. In. VASCONCELOS, R. T. et al. Manual de Saneamento e Proteção Ambiental para os Municípios. Volume II. Belo Horizonte. Escola de Engenharia da UFMG, 1995.

IBGE. ESTATCART: Sistema de Recuperação de Informação Georreferenciadas. Versão2.1. Rio de Janeiro: IBGE, 2004. 1 CD-ROM.

IBGE. Aquidauana. Unidade do Estado de Mato Grosso do Sul. Campo Grande. 2005.

JUNIOR PHILIPPI, A.; MALHEIROS, T. F.; AGUIAR, A. O. Indicadores de Desenvolvimento Sustentável. In. PHILIPPI JÚNIOR, A. Saneamento, Saúde e Ambiente: Fundamentos para um Desenvolvimento Sustentável. Barueri. São Paulo: Manole, Coleção Ambiental; 2. 2005. 
JANNUZZI, P. M. Indicadores sociais no Brasil. 2. Ed. Campinas, SP. Ed. Alínes, 2003.

SANTOS, R. F. Planejamento Ambiental: Teoria e prática. São Paulo. Oficina de Textos, 2004.

SANTOS, Ricardo Miranda dos. A utilização do Indicador de Salubridade Ambiental - ISA como ferramenta de planejamento aplicado à cidade de Aquidauana/MS. 2008. 164 f. Dissertação (Mestrado em Geografia) - Universidade Federal de Mato Grosso do Sul, Campus de Aquidauana/MS. 2008.

SÃO PAULO. ISA - Indicador de Salubridade Ambiental: Manual Básico. Conselho Estadual de Saneamento - CONESAN, Secretária Estadual de Recursos Hídricos Saneamento e Obras. São Paulo. Brasil. 1999. 